\title{
Efecto del bypass gástrico a largo plazo ( 7 a 10 años) en pacientes con obesidad severa y mórbida sobre el peso corporal, diabetes, dislipidemia y desarrollo de anemia
}

Departamento de Cirugía, Hospital Clínico de la Universidad de Chile.

Recibido el 14 de enero de 2011, aceptado el 30 de agosto de 2011.

Correspondencia a:

Dr. Attila Csendes, FACS

Departamento de Cirugía,

Hospital Clínico de la

Universidad de Chile.

Santos Dumont 999,

Santiago, Chile.

Fono: 56-2-7774387.

E-mail: acsendes@ redclinicauchile.cl

\author{
ATTILA CSENDES J., KARIN PAPAPIETRO V., ANA MARÍA BURGOS L., \\ ENRIQUE LANZARINI S., MOIRA CANOBRA L.
}

\section{Results of gastric bypass for morbid obesity after a follow up of seven to 10 years}

Background: There is a paucity of information about the long term effects of gastric bypass for morbid obesity. Aim: To study the evolution of weight and complications of obesity, seven to 10 years after gastric bypass surgery. Material and Methods: One hundred eighteen subjects with morbid obesity, aged 15 to 66 years (103 women), were followed for a mean of 94 months after surgery. Body weight, fasting blood glucose, total cholesterol, triglycerides and hemoglobin were measured before surgery and during follow up. Results: At 24 months of follow up, all patients lost weight and there was a mild weight increase at 94 months, that paralleled the preoperative body mass index. Diabetes, hypercholesterolemia and hypertriglyceridemia subsided in 95, 87 and 94\% of cases, respectively. Twenty percent of patients had mild anemia and 11\% moderate or severe anemia. No patient recovered the preoperative weight. Conclusions: Weight reducing effects of gastric bypass are maintained after 94 months of follow up with the expected health benefits.

(Rev Med Chile 2011; 139: 1414-1420).

Key words: Body mass index; Gastric bypass; Obesity, morbid.
L

a obesidad, definida como un índice de masa corporal mayor a $30 \mathrm{~kg} / \mathrm{m}^{2}$, es una patología

$\checkmark$ de muy alta prevalencia mundial y también en Chile. Datos recientes del Ministerio de Salud ${ }^{1}$ señalan que en sujetos sobre 15 años, la obesidad está presente en $25 \%$. Se observó que 300.000 personas de la población chilena tiene obesidad mórbida, cifra que era de 130.000 el año 2003. El único tratamiento efectivo que logra una baja significativa de peso a largo plazo, comparado con tratamiento médico, es el tratamiento quirúrgico ${ }^{2}$, cuyo "gold standard" es el bypass gástrico ${ }^{3}$. Los resultados postoperatorios de nuestro grupo tanto en términos de mortalidad operatoria, morbilidad, como efecto a 12 y 24 meses de operados han sido ampliamente analizados en varias publicaciones tanto nacionales como en el extranjero ${ }^{4-7}$. Sin embargo, no hay reportes en Chile respecto al comportamiento del peso corporal y comorbilidades en el largo plazo después de bypass gástrico.

El objetivo del presente estudio fue determinar los resultados entre 7 y 10 años de la cirugía, en cuanto a cambios del peso corporal y evolución de algunas comorbilidades metabólicas.

\section{Material y Método}

1. Pacientes estudiados: El grupo comprende a 118 obesos mórbidos operados entre el año 
Seguimiento a 10 años de obesos sometidos a bypass gástrico - A. Csendes et al

2000 y 2003, todos sometidos a un bypass gástrico resectivo por vía laparotómica ${ }^{4,6}$, de un total de 521 pacientes operados en ese período de tiempo. Estos pacientes se seleccionaron de la base de datos que existe en el Departamento de Cirugía, que comenzó en agosto de 1999. Los pacientes fueron contactados a través de llamada telefónica o carta enviada a domicilio, citándolos para un control médico personalizado con uno de los autores (A.C.) No fue posible controlar a todos los operados, ya que con frecuencia cambiaban de domicilio y el Servicio de Registro Electoral ya no entrega el dato preciso de la dirección actual de una persona, por una nueva ley. Esto dificultó enormemente el control de un mayor número de pacientes. También hay un grupo importante que recibió la citación, pero por motivos de trabajo no han concurrido a control hasta ahora. A los pacientes que vinieron a control se les explicó el objetivo del presente estudio tanto por carta como verbalmente, aceptando ser incluidos en esta investigación. En cada uno de ellos se realizó una medición del peso actual, que fue comparado con el peso preoperatorio y el peso a los 24 meses de operado.

2. Evaluación de las comorbilidades metabólicas: A todos los pacientes se le solicitó efectuar un examen sanguíneo, en especial determinando glicemia en ayunas, hemograma, colesterol total y triglicéridos, que se compararon con los resultados del estudio preoperatorio.

3. Cálculo estadístico: Para los cálculos de significación estadística, se realizó la prueba de Fisher tomando un $\mathrm{p}<0,05$ como significativo.

4. Seguimiento: El control clínico y de laboratorio se realizó en promedio a los 94 meses desde la operación, con un rango entre 80 y 120 meses.

\section{Definición de los parámetros:}

a. La diabetes se definió como una hiperglicemia mayor a $126 \mathrm{mg} / \mathrm{dl}$ en ayunas. La remisión se consideró cuando la glicemia basal estuvo bajo $100 \mathrm{mg} \%$ y no se tomaba ningún medicamento.

b. Hipercolesterolemia se consideró a un valor de colesterol total sobre $201 \mathrm{mg} / \mathrm{dl}$.

c. Hipertrigliceridemia se consideró a un valor de triglicéridos sobre $151 \mathrm{mg} / \mathrm{dl}$. d. Anemia se consideró si la hemoglobina era menor o igual a 11,9 g/dl. Se definió como leve un valor entre 10 y $11,9 \mathrm{~g} / \mathrm{dl}$ y moderada o severa un valor bajo $9,9 \mathrm{~g} / \mathrm{dl}$.

6. Calculo de peso: Para los cálculos de la pérdida de exceso de peso, se tomaron los valores normales según peso y talla de la tabla aceptable para adultos del Departamento de Nutrición de la Facultad de Medicina de la Universidad de Chile, datos adoptados de la Sociedad de Actuarios de USA, Metropolitan Life Insurance Company.

\section{Resultados}

El grupo de 118 pacientes controlados en promedio a 94 meses de la operación correspondió a 103 mujeres y 15 hombres. La edad promedio al momento de la cirugía fue para las mujeres de 42,7 años $\pm 11,5$ (rango 19-66) y para los hombres de 42,9 $\pm 12,8$ (rango 15-58), sin diferencias significativas. Considerando el total de pacientes, $38(32,2 \%)$ tenían un IMC entre 35 y $39,9 \mathrm{~kg} / \mathrm{m}^{2}$, $70(59,3 \%)$ tenían un IMC entre 40 y $49,9 \mathrm{~kg} / \mathrm{m}^{2}$ y $10(8,5 \%)$ tenían un IMC igual o superior a 50 $\mathrm{kg} / \mathrm{m}^{2}$. Ningún paciente tenía un IMC menor a $35 \mathrm{~kg} / \mathrm{m}^{2}$ de acuerdo a las normas internacionales de la NIH para indicar la cirugía ${ }^{8}$. Estos pacientes tenían algunas comorbilidades metabólicas presentes al momento de la cirugía, como la diabetes y la dislipidemia como se muestra en la Tabla 1, separado de acuerdo al grado de obesidad. No se observaron diferencias significativas en cuanto a niveles sanguíneos de colesterol total o triglicéridos de acuerdo a la severidad de la obesidad. En la Tabla 2 se señala el IMC antes de la cirugía y en el control actual, mostrando el valor absoluto de disminución del IMC y su \% de descenso. Se aprecia que a mayor grado de obesidad, mayor fue el descenso del IMC. Todos los descensos fueron estadísticamente significativos $(\mathrm{p}<0,0001)$. La Tabla 3 muestra la evolución del peso corporal a los 24 meses de operado y a los 94 meses promedio después de la cirugía, señalando además la variación del peso preoperatorio y a los 24 meses después de la cirugía y a la variación entre los 24 y 94 meses después de la cirugía, según severidad de la obesidad. En todos los grupos se aprecia una disminución muy significativa del peso en el primer control. Sin embargo, en los 3 grupos se 
Tabla 1. Distribución de diabetes y dislipidemia según grado de obesidad $(n=118)$

\begin{tabular}{|c|c|c|c|c|}
\hline & \multicolumn{3}{|c|}{ IMC (Kg/m²) } & \multirow[b]{2}{*}{$\mathbf{p}$} \\
\hline & $\begin{array}{l}35-39,9 \\
n=35\end{array}$ & $\begin{array}{l}40-49,9 \\
n=70\end{array}$ & $\begin{array}{l}>/=\mathbf{5 0} \\
\mathrm{n}=\mathbf{1 0}\end{array}$ & \\
\hline $\begin{array}{l}\text { Diabetes } \\
n=21(17,8 \%)\end{array}$ & $4(10,5 \%)$ & $14(20 \%)$ & $3(30 \%)$ & n.s. \\
\hline $\begin{array}{l}\text { Colesterol > } 201 \mathrm{mg} / \mathrm{d} \\
\mathrm{n}=49(41,5 \%)\end{array}$ & 19 (50\%) & $25(35,7 \%)$ & $5(50 \%)$ & n.s. \\
\hline $\begin{array}{l}\text { Triglicéridos > } 151 \mathrm{mg} / \mathrm{d} \\
\mathrm{n}=53(44,9 \%)\end{array}$ & $15(39,5 \%)$ & $34(48,6 \%)$ & $4(40 \%)$ & n.s. \\
\hline
\end{tabular}

n.s. $=$ No significativo.

Tabla 2. Índice de masa corporal (IMC) antes de la cirugía y en el control actual, según severidad de la obesidad

\begin{tabular}{|c|c|c|c|c|c|}
\hline \multirow{2}{*}{$\begin{array}{l}\text { IMC } \\
\mathrm{kg} / \mathrm{m}^{2}\end{array}$} & \multirow[t]{2}{*}{ Preop } & \multirow{2}{*}{$\begin{array}{l}94 \text { meses } \\
\text { postop }\end{array}$} & \multicolumn{3}{|c|}{ Disminución IMC $\left(\mathbf{k g} / \mathbf{m}^{2}\right)$} \\
\hline & & & $\begin{array}{c}\text { Valor } \\
\text { absoluto }\end{array}$ & $\%$ & $\mathbf{p}$ \\
\hline $35-39,9$ & $37,3 \pm 1,7$ & $26,6 \pm 3,2$ & 10,7 & 28,6 & $<0,0001$ \\
\hline \multicolumn{6}{|l|}{$n=38$} \\
\hline $40-49,9$ & $44,06 \pm 2,7$ & $30,0 \pm 4,2$ & 14,06 & 31,9 & $<0,0001$ \\
\hline \multicolumn{6}{|l|}{$n=70$} \\
\hline$>/=50$ & $53,2 \pm 3,2$ & $33,9 \pm 3,8$ & 19,3 & 36,2 & $<0,0001$ \\
\hline$n=10$ & & & & & \\
\hline
\end{tabular}

observa un aumento de peso entre el $1^{\text {er }}$ y $2^{\circ}$ control, que es proporcional al peso preoperatorio. El porcentaje de pérdida de exceso de peso fue inversamente proporcional al peso preoperatorio observando una disminución de este porcentaje en el $2^{\circ}$ control alejado después de la cirugía que es paralelo al peso preoperatorio. Estos resultados se representan también en las Figuras 1 y 2. En la Tabla 4 se detallan los cambios de peso corporales en controles sucesivos de acuerdo a la severidad de la obesidad. Se aprecia que hubo un aumento de peso que fue progresivamente mayor y paralelo a una obesidad más severa. El número de pacientes que aumentó $5 \mathrm{~kg}$ o más de peso entre el I y II control también fue paralelo a la severidad de la obesidad. El número de pacientes con IMC bajo $30 \mathrm{~kg} /$ $\mathrm{m}^{2}$ en el último control era de $87 \%$ para un IMC preoperatorio entre 35 y $39,9 \mathrm{~kg} / \mathrm{m}^{2}$, de $54 \%$ para un IMC entre 40 y $49,9 \mathrm{~kg} / \mathrm{m}^{2}$ y $20 \%$ para un IMC sobre $50 \mathrm{~kg} / \mathrm{m}^{2}$. Ningún paciente recuperó el peso preoperatorio.

En la Tabla 5 se muestran algunas comorbilidades metabólicas en el

Tabla 3. Evolución del peso corporal antes y 24 y 94 meses después del bypass gástrico según magnitud de la obesidad

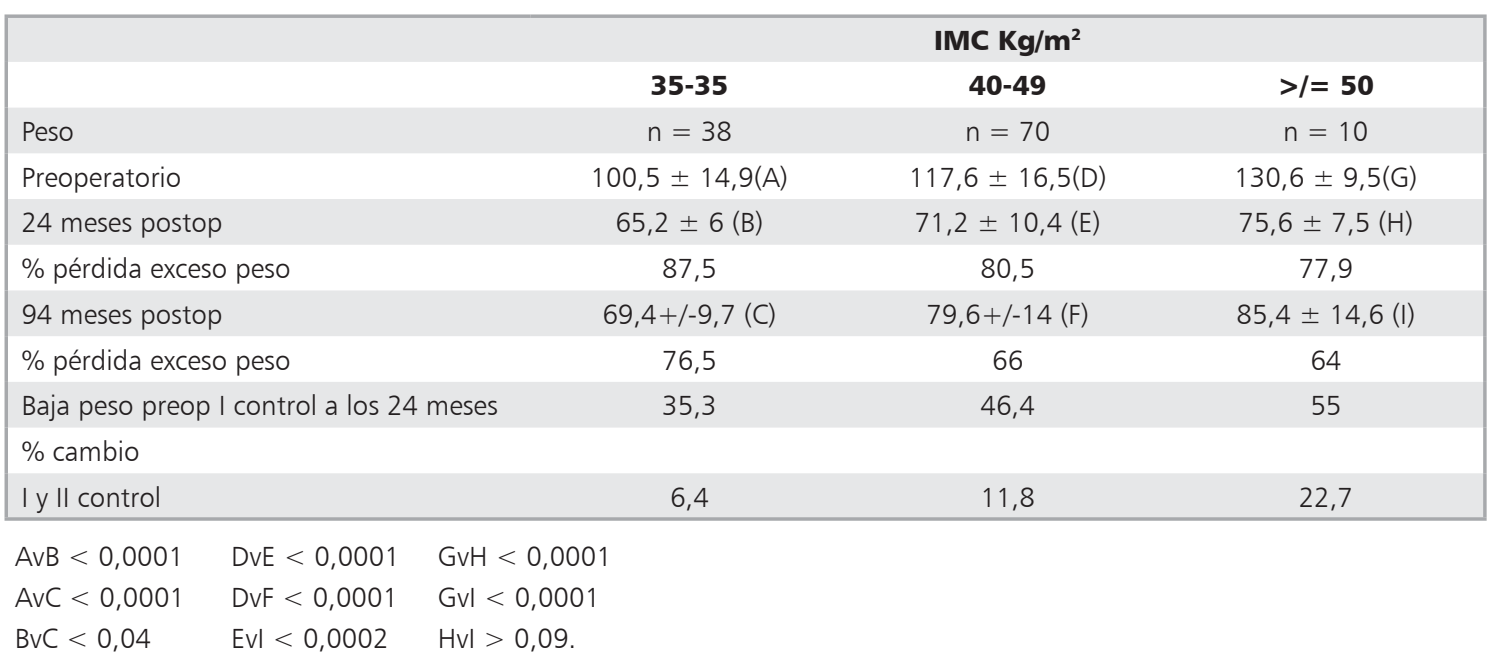



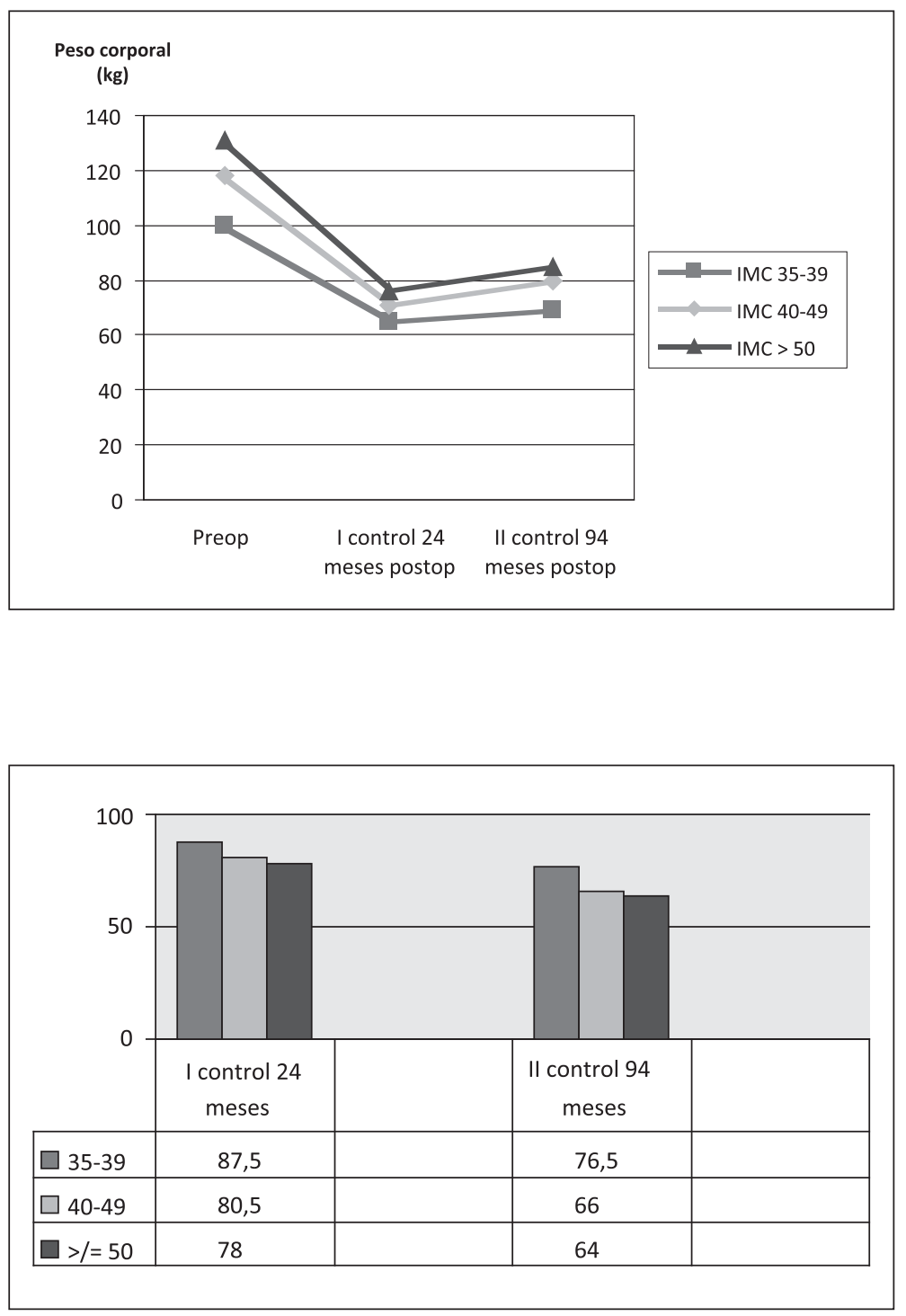

Figura 1. Evolución peso promedio en 2 controles postoperatorios sucesivos.

Figura 2. $\%$ de pérdida del exceso de peso.

Tabla 4. Evolución del peso corporal en controles sucesivos según grado de obesidad

\begin{tabular}{|c|c|c|c|}
\hline & \multicolumn{3}{|c|}{ IMC $\left(\mathbf{k g} / \mathbf{m}^{2}\right)$} \\
\hline & $\begin{array}{c}35-39 \\
n=38\end{array}$ & $\begin{array}{c}40-49 \\
n=70\end{array}$ & $\begin{array}{l}>I=\mathbf{5 0} \\
n=10\end{array}$ \\
\hline - $\mathrm{N}^{\circ}$ pacientes con IMC $<30$ al último control & $33(87 \%)$ & $38(54 \%)$ & 2 (20\%) \\
\hline - $\mathrm{N}^{\circ}$ pacientes que subieron $5 \mathrm{~kg}$ o más entre I y II control & $14(36,8 \%)$ & $44(58,5 \%)$ & $6(60 \%)$ \\
\hline - Aumento de peso en kg entre I y II control & 4,2 & 8,4 & 10 \\
\hline - $N^{\circ}$ pacientes que recuperan peso preoperatorio & 0 & 0 & 0 \\
\hline
\end{tabular}


Tabla 5. Evolución de comorbilidades metabólicas en el control alejado a 94 meses

\begin{tabular}{|c|c|c|c|c|}
\hline & & Preop & Control alejado & $\mathbf{p}$ \\
\hline \multicolumn{5}{|c|}{ 1.- Diabetes (Glicemia > $126 \mathrm{mg} \%) \quad(\mathrm{n}=21)$} \\
\hline$x=$ & & $155 \pm 28,7$ & $104,6 \pm 48$ & $<0,0005$ \\
\hline Mediana & & 147 & 92 & \\
\hline Rango & & $130-238$ & $78-292$ & \\
\hline \multicolumn{5}{|c|}{ 2.- Hipercolesterolemia $>201 \mathrm{mg} \% \quad(n=49)$} \\
\hline$x=$ & & $238 \pm 32,3$ & $179,8 \pm 30,9$ & $<0,0001$ \\
\hline Mediana & & 227 & 179 & \\
\hline Rango & & $202-327$ & $100-255$ & \\
\hline \multicolumn{5}{|c|}{ 3.- Hipertrigliceridemia $>151 \mathrm{mg} \% \quad(n=53)$} \\
\hline $\mathrm{x}=$ & & $236 \pm 105$ & $116,8 \pm 45,1$ & $<0,0001$ \\
\hline Mediana & & 211 & 104 & \\
\hline Rango & & $153-812$ & $30-262$ & \\
\hline 4.- Anemia $\mathrm{HB}<12 \mathrm{~g} / \mathrm{dl}$ & $(n=37)$ & & & \\
\hline $10-11,9 \mathrm{~g} / \mathrm{dl}$ & $n=24$ & $11,2 \pm 0,5(10-11,9)$ & & \\
\hline$<9,9 \mathrm{~g} / \mathrm{d}$ & $\mathrm{n}=13$ & $8,2 \pm 1,09(6,7-9,7)$ & & \\
\hline
\end{tabular}

control alejado. De los 21 pacientes diabéticos, hubo normalización de los niveles de glicemia en 20 pacientes $(95 \%)$, y ninguno tomaba medicamentos. Sólo un paciente se mantenía con niveles altos de glicemia, a pesar de haber bajado de un IMC de 46,7 a $38 \mathrm{~kg} / \mathrm{m}^{2}$. Hubo un paciente en el que apareció diabetes al control alejado, teniendo una glicemia preoperatoria de $85 \mathrm{mg} /$ dl que subió a $131 \mathrm{mg} / \mathrm{dl}$, con una baja del IMC de 46 a $29 \mathrm{~kg} /$ $\mathrm{m}^{2}$. De los 49 pacientes con hipercolesterolemia, hubo una normalización de los niveles de colesterol sérico en 43 (87,7\%), 3 pacientes mantuvieron niveles similares al preoperatorio y 3 pacientes $(6,1 \%)$ tuvieron niveles mayores comparado al estudio preoperatorio. De los 53 pacientes con hipertrigliceridemia, estos niveles se normalizaron en 50(94,3\%), se mantuvieron igual en 1 paciente y se elevaron en 2 pacientes $(3,9 \%)$ a pesar de haber descendido el IMC de 44 a 34 y de 49 a $36 \mathrm{~kg} / \mathrm{m}^{2}$ en ambos. La anemia se presentó en 37 pacientes $(31,3 \%)$ siendo leve en $24(20,3 \%)$ y moderado a severo con HB bajo $10 \mathrm{~g} /$ día en 13 pacientes (11\%). Todos se trataron con fierro y ácido fólico, ya que en todos la anemia era microcítica e hipocroma. Hernia incisional se presentó en 26 pacientes $(22 \%)$, siendo operados por esta patología hasta la fecha 20 pacientes.

\section{Discusión}

Los resultados del presente estudio sugieren que: 1) en todos los pacientes con obesidad severa y mórbida sometidos a bypass gástrico, hay una disminución significativa del peso corporal después de la cirugía; 2) esta baja de peso es mayor en los pacientes con mayor severidad de la obesidad; 3) que hay un aumento de peso a medida que el control sea más alejado; 4) la diabetes se controló en 95\% de los casos; 5) la hipercolesterolemia se normalizó en $88 \%$, la hipertrigliceridemia en $94 \%$; 6) hay anemia en $31 \%$ de los pacientes.

En nuestro análisis sólo hemos tomado la evaluación de algunos de los parámetros más importantes en pacientes con obesidad mórbida. No hemos evaluado otros síntomas como apnea de sueño, osteoarticulares, reflujo gastroesofágico, hipertensión arterial, etc., que han sido motivo de otras publicaciones referentes a control alejado de estos obesos operados 9 . Sin embargo, casi todos se refieren a la evaluación 12 a 24 meses desde la operación $^{4,6,7,9}$, pero no hay referencias de control alejado de 5 años o más en nuestro país. Por esto que pretendimos evaluar los resultados del bypass gástrico hasta 10 años después de la cirugía, tal 
Seguimiento a 10 años de obesos sometidos a bypass gástrico - A. Csendes et al

como se han publicado en algunos reportes internacionales.

Lo primero que llama la atención es que todos los pacientes presentan una baja de peso variable, dependiendo de la magnitud del peso preoperatorio y que ninguno de los pacientes ha recuperado su peso original hasta por lo menos 8 a 10 años después de la cirugía. El máximo descenso de peso se observó a los 24 meses desde la operación, pero en todos los grupos, se aprecia un aumento de peso en el control alejado, que es paralelo y proporcional al grado de la obesidad. Nuestro grupo está evaluando los aspectos nutricionales y psicológicos de estos pacientes en el largo plazo después de la cirugía, que será motivo de otra publicación, ya que hay profundos cambios psicológicos y de hábitos de comer en estos pacientes.

Llama la atención que en publicaciones internacionales hay muy pocos reportes referentes a la evolución del peso a largo plazo (10 o más años) después de cirugía bariátrica. El primer trabajo publicado en 1995 por Pories et al ${ }^{10}$ demostró que los pacientes sometidos a bypass gástrico presentaron $70 \%$ de pérdida del exceso de peso a los 2 años de operado, que disminuyo a 50\% a los 14 años de operado. El segundo estudio del año 1997 por MacDonald et $\mathrm{al}^{11}$ en 154 pacientes sometidos a bypass gástrico, demostró un \% de pérdida del exceso de peso a los 2 años de $65 \%$, que a los 14 años fue de $60 \%$. Posteriormente los estudios del Grupo Sueco de Obesidad ${ }^{12,13}$ demostraron claramente los mismos efectos del bypass gástrico en 2.010 obesos mórbidos: una pérdida de peso máxima a los 2 años de operado de $32 \%$ del peso preoperatorio, que a los 10 años de operado se estabilizó en un porcentaje de pérdida de peso de $25 \%$, llegando a una cifra cercana del $27 \%$ a los 15 años de operado: Es decir, todos los estudios coinciden con nuestros resultados, en el sentido que 8 a 10 años después de la cirugía se aprecia un aumento de peso comparado con lo observado 1 a 2 años postoperatorio, estabilizándose a los 10 años y no variando a los 15 años de operado, pero que nunca alcanza los valores preoperatorios.

El otro aspecto de gran importancia en el presente estudio se refiere a la evolución de algunas comorbilidades, metabólicas, que fueron evaluadas en $100 \%$ de los controlados a largo plazo. La remisión de $95 \%$ de la diabetes en estos pacientes es destacable y lo notable es que se mantiene en el tiempo. Nuestro grupo ya había publicado resulta- dos en diabéticos a 12 y 24 meses de operados, con una revisión de la diabetes, definida como glicemia en ayunas normal y no uso de medicamentos, en $92 \%{ }^{4,9}$. Estudios americanos muestran que esta remisión de la diabetes es muy precoz ${ }^{10,11,14}$, antes que ocurra una baja de peso importante y se mantiene por lo menos hasta 15 años después de la operación ${ }^{10,11}$. Sin embargo, al igual que lo que ocurre con el peso, los estudios suecos sugieren ${ }^{12}$ que a largo plazo se aprecia un leve aumento de la diabetes en los pacientes operados a los 10 años comparado con el resultado a 2 años (7\% versus $1 \%$ respectivamente). No es el propósito del presente estudio analizar los mecanismos de producción y remisión de la diabetes, que serán publicados en corto plazo.

Otro hallazgo significativo fue que $88 \%$ de los pacientes con hipercolesterolemia y $94 \%$ de los pacientes con hipertrigliceridemia mostraron normalización de los otros valores preoperatorios en el control a largo plazo, sin tomar ningún medicamento. Es muy probable que la disminución de la masa grasa libere menos ácidos grasos y por lo tanto, los niveles de lípidos elevados se normalizan y lo más importante es que se mantienen en el tiempo.

Es un hecho conocido y descrito por numerosos autores y también por nuestro grupo ${ }^{9,15}$ que un porcentaje importante, cercano al 30\%, desarrolla un grado variable de anemia por disminución de la absorción de Fe, debido al bypass gástrico. El fierro se absorbe principalmente en el duodeno y yeyuno proximal y al existir este bypass, su absorción disminuye. Sin embargo, con un tratamiento médico prolongado, se logra controlar adecuadamente este trastorno metabólico.

La limitación más importante del presente estudio fue la dificultad para controlar a los pacientes a largo plazo. Es conocido por todos los grupos que se dedican al manejo de pacientes con obesidad severa y mórbida, el enorme esfuerzo que significa citarlos a control.

Este es un punto que ha sido imposible de resolver. Sin embargo, en el presente estudio se ha incluido el mayor número de pacientes posibles de controlar y suponemos que estos datos se pueden extrapolar al grupo completo.

En resumen, el presente estudio demuestra por primera vez en Chile, que el bypass gástrico logra una baja significativa de peso que se recupera parcialmente a largo plazo (entre 7 a 10 años después 
de la cirugía), pero ningún paciente llega a tener el nivel de obesidad preoperatoria. La diabetes en este grupo remitió en 95\%, la hipercolesterolemia en $88 \%$ y la hipertrigliceridemia en $94 \%$. Anemia se observó en $31 \%$ de los pacientes. El bypass gástrico por lo tanto, es una excelente alternativa de tratamiento para pacientes con obesidad severa y mórbida.

\section{Referencias}

1. Ministerio de Salud, Departamento de Estadísticas. Octubre 2010.

2. Csendes A, Burdiles P, Papappietro K, Burgos AM. Comparación del tratamiento médico y quirúrgico en pacientes con obesidad grado III (obesidad mórbida). Rev Med Chile 2009; 137: 559-66.

3. Csendes A, Korn O, Burdiles P. Historia de la cirugía bariátrica en Chile (1986-2005). Rev Chile Cir 2006; 58: 386-9.

4. Csendes A, Burdiles P, Papapietro K. Results of gastric bypass plus resection of the distal excluded segment in patients with morbid obesity. J Gastroint Surg 2005; 9: 121-31.

5. Csendes A, Maluenda F. Morbimortalidad de la cirugía bariátrica. Experiencia chilena en 10 instituciones de salud. Rev Chile Cir 2006; 58: 208-12.

6. Csendes A, Burdiles P, Burgos AM, Díaz JC, Braghetto I, Maluenda F, et al. Riesgo perioperatorio del bypass gástrico reseccional en pacientes con obesidad mórbida. Estudio prospectivo de 684 pacientes. Rev Med Chile 2006; 134: 849-54.

7. Carrasco F, Papapietro K, Csendes A, Salazar G, Echeñique $\mathrm{C}$, Lisboa $\mathrm{C}$, et al. Changes in resting energy expen- ditive and body composition after weight loss following Roux-en-Y gastric bypass. Obes. Surg. 2007;17:608-616.

8. NIH Gastrointestinal surgery for severe obesity. National Institute of Health Consensus. Development conference. Obes Surg 1991; 1: 257-66.

9. Papapietro K, Díaz E, Csendes A, Díaz JC, Braghetto I, Burdiles P, et al. Evolución de comorbilidades metabólicas asociadas a obesidad después de cirugía bariátrica. Rev Med Chile 2005; 133: 511-6.

10. Pories WJ, Swanson MS, MacDonald KG, Long SB, Morris PG, Brown BM, et al. Who would have thought it? An operation proves to be the most effective therapy for adult onset diabetes mellitus. Ann Surg 1995; 222: 339-50.

11. MacDonald KG, Long SD, Swanson MS, Brown BM, Morris P, Dohm GL, et al. The gastric bypass operation reduces the progression and mortality of non-insulin dependent diabetes mellitus. J Gastroint Surg 1997; 1: 213-20.

12. Sjöström L, Lindroos AK, Peltonen M, Torgerson J, Bouchard C, Carlsson B, et al. Swedish Obese Subjects Study Scientific Group. Lifestyle, diabetes and cardiovascular risk factors 10 years after bariatric surgery. N Engl J Med 2004; 351: 2683-93.

13. Sjöström L, Narbro K, Sjöström CD, Karason K, Larsson B, Wedel H, et al. Swedish Obese Subjects Study. Effects of bariatric surgery on mortality in Swedish obese subjects. N Eng J Med 2007; 23: 357: 741-52.

14. Buchwald H, Avidor Y, Braunwald E, Jensen MD, Pories W, Fahrbach K, et al. Bariatric surgery: a systematic review and meta analysis. JAMA 2005; 293: 1728.

15. Mizón C, Díaz M, Csendes A, Carrasco F, Rebolledo A, Codoceo J, et al. Persistent anemia after Roux-en-Y gastric bypass. Nutrition 2007; 23: 277-80. 